\title{
Utility-Based Video Quality Model in Wireless Ad Hoc Network
}

\section{Yang Tao1, Wenxuan Zang2 \& Qianjin He2}

\section{School of Communication and Information Engineering, University of Posts and} Telecommunications, Chongqing, 400065, China.

2School of Computer Science and Technology, University of Posts and Telecommunications, Chongqing, 400065, China.

Keywords: Ad Hoc, quality, video, model.

\begin{abstract}
At present, domestic and international research on the video transmission mainly concentrated in video coding, multipath transmission, multicast transmission. The transmission and QoS security technology will be studied to against the characteristics of video transmission and wireless ad hoc network.How to transfer more video streaming under conditions of the limited network resource is a meaningful topic.In this paper,the conception of user level into service classification is introduced and a framework named UVQM is proposed.The UVQM can provide QoS security for video transmission and ensure more streaming transmitted without congestion.
\end{abstract}

\section{Introduction}

The video transmission technology used in the wireless ad hoc network solves the problem that how to achieve the efficient transmission of video,but it ignores the QoS support that the network can provide for the transmission. The research on QoS model is from the perspective of network,but it doesn't propose a complete application solution for the video transmission.[1]The existing QoS models can not solve the problem that how to implement the high quality video interaction between more nodes[2] under conditions of the limited network resource.

Therefore,it is very meaningful to propose a video quality model of wireless ad hoc network that not only can satisfy the minimum requirements of video transmission,but also can implement the video interaction between more nodes.

\section{Traffic Differentiation}

Table 1: Priority of traffic

\begin{tabular}{|c|c|c|}
\hline $\begin{array}{c}\text { DCSP } \\
\text { Value }\end{array}$ & $\begin{array}{c}\text { Traffic } \\
\text { Class }\end{array}$ & Priority \\
\hline 100001 & H-IC & 1 \\
\hline 010001 & M-IC & 2 \\
\hline 001001 & L-IC & 3 \\
\hline 100100 & H-CC & 4 \\
\hline 010010 & M-SC & 5 \\
\hline 001010 & L-SC & 6 \\
\hline 010100 & M-CC & 7 \\
\hline 001100 & L-CC & 8 \\
\hline 100010 & H-SC & 9 \\
\hline 100000 & H-BC & 10 \\
\hline
\end{tabular}




\begin{tabular}{|l|l|l|}
\hline 010000 & M-BC & 11 \\
\hline 001000 & L-BC & 12 \\
\hline
\end{tabular}

According to the degree of delay-sensitive,the traffics are divided into 4 classes by 3GPP: Conversational Class(CC), Streaming Class(SC), Interactive Class(IC) and Background Class(BC), their degrees of delay-sensitive are in descending order.The Differentiated Services(DiffServ) model[3,4] uses the front 6 bits of service type identification field in the IP packet header to classify the packets, and these 6 bits are called DSCP value[5]. According to the importance of users, the users are divided into 3 levels:High-level,Middle-level,Low-level. All users can send CC, SC, IC, BC traffics.Introduce the user level and change the format of DSCP value, use the first 3 bits of DSCP value to distinguish the user level: 100(High-level), 010(Middle-level), 001(Low-level); the spare 3 bits are used to distinguish the class of traffic:100(CC),010(SC), 001(IC),000(BC).

According to the experience,we prioritize these traffics as shown in Table 1.

\section{Bandwidth estimation and management}

Based on the IEEE802.11 MAC layer protocol,node can calculate the channel utilization by monitoring the channel busy status[6,7],also can use the channel capacity and utilization to calculate the available bandwidth[8]. The channel utilization $R$ during the sampling period $T$ can be obtained by the following formula:

$$
R=\frac{\text { Busy Time }}{T}
$$

In order to reduce the error of estimation,introducing the smoothing factor $\alpha \in[0,1]$,then obtain the current channel utilization $R_{t}$ by the following formula:

$$
R_{t}=\alpha R_{t-1}+(1-\alpha) R \quad R_{t} \in[0,1]
$$

The available bandwidth of a node at time $t$ is:

$$
W_{t}=\beta C\left(1-R_{t}\right) \quad \beta \in(0,1)
$$

$C$ is the channel capacity (IEEE 802.11 radio channel capacity is $2 \mathrm{Mbps}$ ), $\beta$ is environmental impact factor.

Table 2: Bandwidth usage information of the streaming

\begin{tabular}{|c|c|c|c|}
\hline Streaming & $\begin{array}{c}\text { Traffi } \\
\text { c } \\
\text { Class }\end{array}$ & $\begin{array}{c}\text { Bandwidth } \\
\text { (bps) }\end{array}$ & $\begin{array}{c}\text { Minimum requirement } \\
\text { bandwidth (bps) }\end{array}$ \\
\hline$s_{1}$ & H-CC & $x_{1}$ & $x_{1}^{\text {min }}$ \\
\hline$s_{2}$ & M-SC & $x_{2}$ & $x_{2}^{\text {min }}$ \\
\hline$s_{3}$ & L-SC & $x_{3}$ & $x_{3}^{\text {min }}$ \\
\hline
\end{tabular}

The traffic transmission rate is between the the minimum and maximum bandwidth requirements.Here the concept of stretch bandwidth is introduced to represent the difference between the traffic transmission rate and the smallest value of all traffic minimum bandwidth requirements.Satisfy the traffic minimum bandwidth requirement and release the stretch bandwidth,so that the stretch bandwidth can be considered as available bandwidth,thus the data transmission during the time that lack of network resource is possible.Establish the bandwidth information table to record the bandwidth usage information of the streaming that flowing through the node.As shown in Table 2. 


\section{Admission Control}

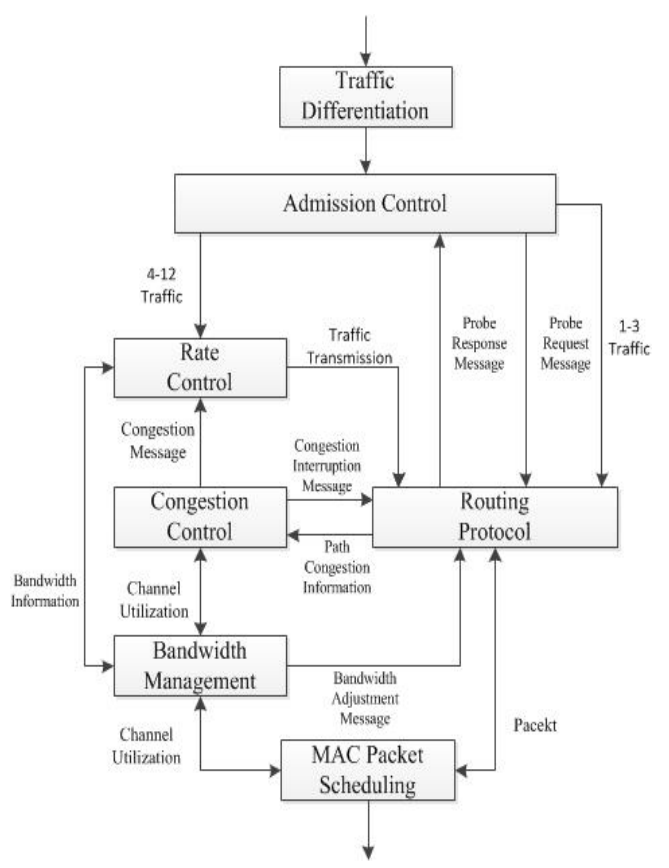

Fig. 1 Admission control process

When the source node receives the transmission request,it determines whether there are enough network resources to ensure the traffic transmission by the admission control module.Admission control module's workflow is as follows:

Step1:The traffic initiates a transmission request,first of all, the traffic needs to be classified by the traffic differentiation module,then do different admission control for different traffic classes.1-3 traffics can be directly transmitted,4-9 traffics need to send a probe request packet to find a path that meet the minimum bandwidth requirements,10-12 traffics can be sent directly by the rate control module.

Step2:Admission control module first determines whether the source node's assignable bandwidth satisfies the minimum bandwidth requirements of the traffic, the assignable bandwidth includes the available bandwidth and stretch bandwidth.If satisfied, sends a probe request packet to the destination node to obtain the information of available bandwidth on the path, the probe request packet contains the traffic class field and minimum bandwidth requirement field.If not, then determines whether the lower priority traffic's sum of minimum available bandwidth and assignable bandwidth satisfies the bandwidth requirements,if satisfied, sends a probe request packet to the destination node, if not,refuses the traffic to access.

Step3: When the intermediate node receives the probe request packet, it needs to get the traffic class field and minimum bandwidth requirement field to determine whether it's assignable bandwidth satisfies the minimum bandwidth requirements.If satisfied,forwarding the probe request packet directly.If not, then determines whether the lower priority traffic's sum of minimum available bandwidth and assignable bandwidth satisfies the bandwidth requirements, if satisfied, forwarding the probe request packet to the destination node,if not,sends a reply that the source node refuses the traffic to access.

Step4:After the destination node receives the probe request packet, it needs to determine whether it's bandwidth satisfies the minimum bandwidth requirements of the traffic.If satisfied, sends a probe response packet to the source node to response the probe request packet, then add the traffic class and minimum bandwidth requirements to the bandwidth information table.If need the traffics in the bandwidth information table to release the stretch bandwidth,start from the lowest priority traffic until it meets the minimum bandwidth requirements.If still not enough,interrupt the traffic in the same way until it meets the minimum bandwidth requirements.

Step5:After the intermediate node receives the probe response packet, it also adds the traffic information in the bandwidth information table, and takes a series of measures to provide adequate 
bandwidth for the access of the new traffic.

Step6:After the source node receives the probe response packet,it adds the traffic information in the bandwidth information table,then accept the traffic transfer request and start sending data.

\section{Congestion Control}

The network congestion that caused by the interference of the wireless link or the change of network topology is unavoidable. When a node perceived the network congestion,first of all,it checks it's bandwidth information table,then sends the congestion notification(such as ECN message ) to the lowest priority traffic,informs the source node to reduce the transmission rate and release the stretch bandwidth.If not enough,keep sending the congestion notification to the higher priority traffic.If the congestion still exist after all the stretch bandwidths have been released,sends the congestion interrupt notification in the same way until the congestion has been eliminated.After the traffic transmission is interrupted, the source node can re-initiate a probe request packet to find a path that meets the minimum bandwidth requirements.

\section{Utility-Based Video Quality Model}

On the basis of these studies, this paper presents a utility-based video quality model, referred UVQM, while satisfying the minimum requirements of video transmission, to achieve the video interaction between more nodes. The description of UVQM node’s behaviors as shown in Fig 1.

The objective function of UVQM can be expressed as:

$$
U_{N}=\alpha T+\beta C_{v s}
$$

$U_{N}$ represents the network utility, $T$ represents the network throughput, $C_{v s}$ represents the number of the video streams in the network, $\alpha$ and $\beta$ are impact factors. This function means that in the networks that with the same throughput ,the more video streaming in the network, the network utility is better.

\section{Simulation}

\subsection{Simulation Environment}

Table 3: Basic simulation environment parameters

\begin{tabular}{|c|c|}
\hline The simulation parameters & The parameter value \\
\hline Routing protocol & AODV \\
\hline Area (m2) & $1000 \times 1000$ \\
\hline Number of nodes & 32 \\
\hline Transmission range (m) & 250 \\
\hline The bandwidth (Mbps) & 2 \\
\hline The MAC layer protocol & IEEE802.11 \\
\hline Mobile model & Random waypoint mobility \\
\hline Node movement speed \\
(m/s)
\end{tabular}




\begin{tabular}{|c|c|}
\hline Simulation time(s) & 900 \\
\hline
\end{tabular}

In the area of $1000 \mathrm{~m}$ by $1000 \mathrm{~m}, 32$ nodes are randomly distributed, and each node's communication range is $250 \mathrm{~m}$. Randomly select 2 nodes as H-level users, 10 nodes as M-level users, 20 nodes as L-level users from the 32 nodes.The rates of CC,SC traffics are[30,100] Kbps and $[128,300]$ Kbps.Loop in this order every 10 seconds : H, M, M, L, L, L to select a node sends CC / SC traffics to any node.

\subsection{Simulation Result}

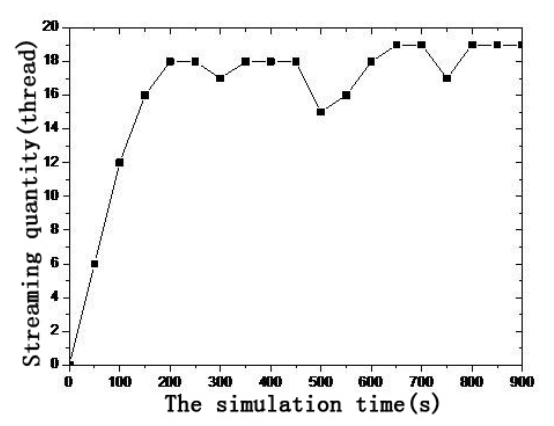

Fig. 2 CC/SC streaming quantity

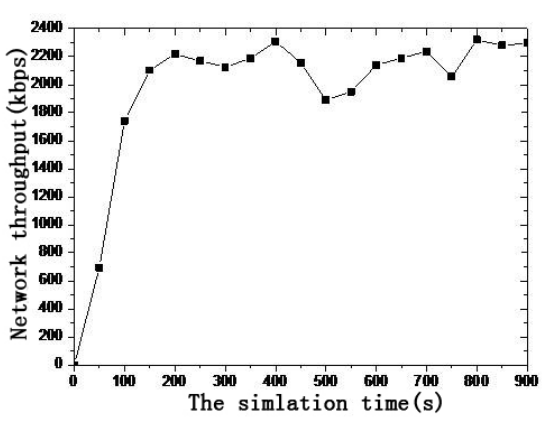

Fig. 3 Network throughput

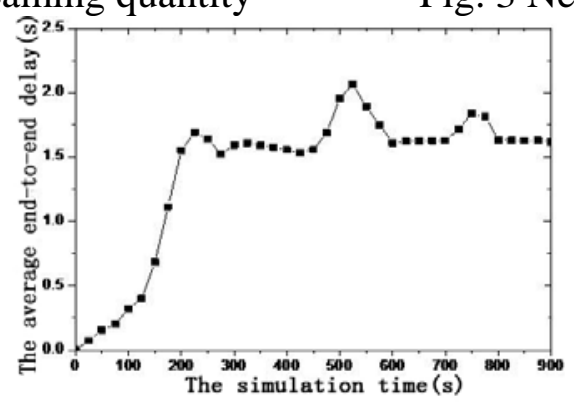

Fig. 4 Average end-to-end delay

It can be seen from the figure 2, within a period of time after the start of the simulation,the quantity of CC / SC streaming is increasing,then fluctuate within a certain range after the 200s, because after the quantity of streaming reaches a certain extent, when a new transmission request arrives, the source node needs to determine whether to accept the streaming through the admission control module.At the time of 500s, 750s there are substantial fluctuations,because at those time,the network topology changes, the path is interrupted,so the node has to release the network resource that is occupied by the streaming and find a new available path.

It can be seen from the figure 3, with the growth of the CC / SC streaming quantity, the throughput shows a rising trend.At the time of 150 s the throughput has nearly reached the maximum,but the quantity of CC / SC streaming has not reached the maximum,because the congestion control module changes the rate of the traffics to increase the network utility.

It can be seen from the figure 4,with the growth of the CC / SC streaming quantity,the average end-to-end delay keeps increasing. At the time of 200s,the quantity of streaming has nearly reached the maximum,the average end-to-end delay is also stable,because the admission control mechanism and congestion control mechanism together to ensure the load does not exceed the tolerance range of the network and guarantee the minimum bandwidth requirements are satisfied.At the time of 450s and 700s,there are substantial changes,we can infer that the topology of network has changed and resulted in the increasing of the average end-to-end delay.

\section{Conclusion}

Overall, the UVQM can effectively control the quantity of CC / SC streaming in the network, and prevent a network congestion or paralysis that caused by the excessive sending.The UVQM can 
ensure more CC / SC streaming while maintain a high network throughput,it also can protect the transmission of CC / SC streaming and make sure the end-to-end delay in the acceptable range.

\section{References}

[1] Xiao Hannan, W. K. G. Seah, Kee Chaing Chua. A flexible quality of service model for mobile ad-hoc networks[C]. 2000 IEEE 51st Vehicular Technology Conference Proceedings. 2000. 1:445-449.

[2] Zhao Yu-ting, Dai Guan-zhong, Mu De-jun. Performance Evaluation of Real-Time Video over Wireless ad hoc Networks[C]. Seventh International Conference on Grid and Cooperative Computing. 2008. 653-657.

[3] Xiao Hannan, Kee Chaing Chua, W. Seah, A. Lo. On service prioritization in mobile ad-hoc networks[C]. IEEE International Conference on Communications. 2001. 6:1900-1904.

[4] Gahng-Seop Ahn, A. T. Campbell, A. Veres, Li-Hsiang Sun. SWAN:service differentiation in stateless wireless ad hoc networks[C]. Twenty-First Annual Joint Conference of the IEEE Computer and Communications Societies. 2002. 2:457-466.

[5] S. Blake. An architecture for Differentiated Services. IETF RFC2475, 1998.

[6] S. Firouzabadi, D. C. O'Neill, A. Goldsmith. Distributed wireless network utility maximization[C]. 44th Annual Conference on Information Sciences and Systems. 2010. 1-6.

[7] Bin Li, A. Eryilmaz, Ruogu Li. Wireless scheduling for network utility maximization with optimal convergence speed[C]. 2013 Proceedings IEEE INFOCOM. 2013. 2112 - 2120.

[8] Qiulin, Song Meina, Wei Jin. Utility optimal multimedia communications with QoS guarantees in cooperative networks[C]. 2010 IEEE 2nd Symposium on Web Society. 2010. 270-276. 